\title{
Quantifying the effect of sea level rise and flood defence - a point process perspective on coastal flood damage
}

\author{
M. Boettle ${ }^{1}$, D. Rybski ${ }^{1}$, and J. P. Kropp ${ }^{1,2}$ \\ ${ }^{1}$ Potsdam Institute for Climate Impact Research (PIK), Potsdam, Germany \\ ${ }^{2}$ University of Potsdam, Institute of Earth and Environmental Science, Potsdam, Germany \\ Correspondence to: D. Rybski (ca-dr@rybski.de) \\ Received: 17 August 2015 - Published in Nat. Hazards Earth Syst. Sci. Discuss.: 13 October 2015 \\ Revised: 18 January 2016 - Accepted: 22 January 2016 - Published: 29 February 2016
}

\begin{abstract}
In contrast to recent advances in projecting sea levels, estimations about the economic impact of sea level rise are vague. Nonetheless, they are of great importance for policy making with regard to adaptation and greenhouse-gas mitigation. Since the damage is mainly caused by extreme events, we propose a stochastic framework to estimate the monetary losses from coastal floods in a confined region. For this purpose, we follow a Peak-over-Threshold approach employing a Poisson point process and the Generalised Pareto Distribution. By considering the effect of sea level rise as well as potential adaptation scenarios on the involved parameters, we are able to study the development of the annual damage. An application to the city of Copenhagen shows that a doubling of losses can be expected from a mean sea level increase of only $11 \mathrm{~cm}$. In general, we find that for varying parameters the expected losses can be well approximated by one of three analytical expressions depending on the extreme value parameters. These findings reveal the complex interplay of the involved parameters and allow conclusions of fundamental relevance. For instance, we show that the damage typically increases faster than the sea level rise itself. This in turn can be of great importance for the assessment of sea level rise impacts on the global scale. Our results are accompanied by an assessment of uncertainty, which reflects the stochastic nature of extreme events. While the absolute value of uncertainty about the flood damage increases with rising mean sea levels, we find that it decreases in relation to the expected damage.
\end{abstract}

\section{Introduction}

Considering current $\mathrm{CO}_{2}$ emission pathways, severe climate change impacts need to be anticipated (IPCC, 2007; Nicholls and Cazenave, 2010). As one of the most perceivable effects of global warming, sea level rise will amplify the magnitude as well as the frequency of coastal floods (Rahmstorf and Coumou, 2011; Seneviratne et al., 2012) and is likely to have significant economic impacts (Hinkel et al., 2014; Nicholls and Tol, 2006). Even in the case of temperature stabilisation, sea levels will continue to rise for many decades (Meehl et al., 2012). Accordingly, greenhouse-gas mitigation alone will not be sufficient, and additional preventive measures need to be considered to cope with the consequences (Petherick, 2012). The most common method to assess the efficiency of such measures is Cost-Benefit Analysis (Tol, 2002), in which the benefits in terms of averted damage are compared to the investment costs. For this purpose, a concise assessment of potential economic consequences is indispensable.

Adverse effects from sea level rise are particularly expected from storm surges, presupposing the coincidence of extreme tidal and storm conditions (Woodworth et al., 2011). Accordingly, not the mean sea level itself but rather its effect on the tail of the sea level distribution needs to be studied. Since the actual distribution of sea levels is in general unknown, extreme value theory is commonly employed in order to characterise extreme events by a unifying tail distribution (Hawkes et al., 2008).

For estimating the annual flood damage at a specific site (that is the sum of all damages caused within a year), information on the occurrence of flood events, their magnitude 
(b) Sea level rise (c) Flood defence

(a) Extreme sea levels

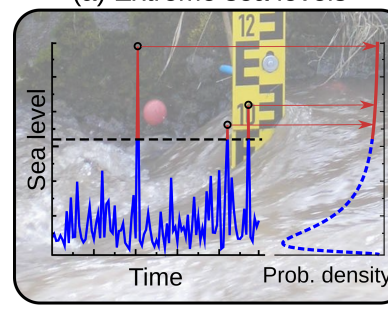

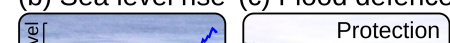

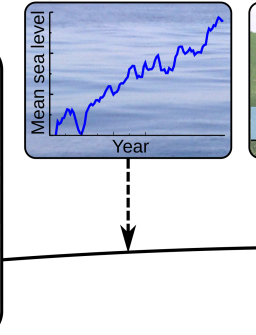

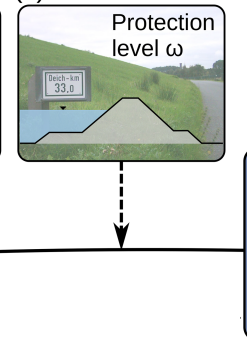

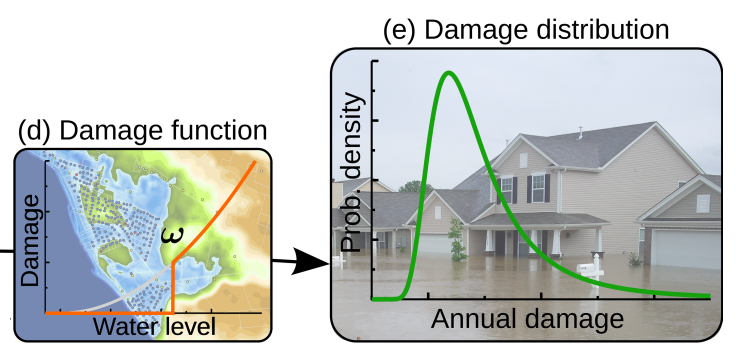

Figure 1. From extreme sea levels to damage. (a) The analysis of extreme sea levels provides parameter estimations for the generalised Pareto distribution. (b) The distribution of sea levels is influenced by mean sea level rise. (c) Flood defence measures, such as dykes, set the threshold below which any damage is prevented. (d) The distribution of extreme sea levels is combined with the corresponding damage via a damage function, providing the total damage in the region under study at a certain maximum flood level. (e) From the resulting distribution of total annual damage the expected annual damage and its standard deviation can be derived. (Photographs: "Ilmpegel Ilmenau" by Michael Sander (2006), "Sea" by Dedda71 (2008), "Kilometermarkierung Deich" by Georg HH (2006), and "Nashville Flood" by Eric Hamiter (2010) from Wikimedia Commons - CC:BY-SA.)

as well as the corresponding damage is required. Due to the stochastic nature of extreme events, the annual damage cannot be predicted for a specific year and is characterised by its average value over a longer time period. In reality, the actual damage fluctuates around this expected annual damage with a certain variability. For instance, there are years without any damage and others where a very unlikely flood event (e.g. a 100- or 1000-year event) occurs. This variability can be measured by several means such as the skewness of the distribution or specific quantile values. We will use the standard deviation for this purpose as it can be straightforwardly derived and further provides an intuitive way to quantify the uncertainty of our damage estimations. Since environmental as well as climatic changes alter the statistics of extreme events, we assume non-stationarity and investigate the development of damage for specific parameter scenarios.

Considering sea level rise, we find analytic relations describing the damage for asymptotic parameter values (i.e. for very large changes) and show that they represent good approximations for the behaviour of damage under current conditions. Furthermore, studying the mitigation effects due to coastal protection measures in an analogous way, we provide three potential decays of residual damage, depending on the shape of the sea level distribution. In general, our analytical relations are capable of describing the development of damage for all parameter variations.

The paper is organised as follows. Section 2 provides the methodologies for the estimation of annual flood damage via a point process. The effect of sea level rise is investigated in Sect. 3, where we provide analytical expressions describing the asymptotic behaviour in a general setting. The generic form of these results allows their application to arbitrary regions, which is exemplified by two case studies in Denmark (Sect. 4). Section 5 investigates the effect of coastal protection in a similar manner. A complementary block maxima approach using the Generalised Extreme Value (GEV) dis- tribution was followed previously (Boettle et al., 2013), in which exactly one - namely the most severe - flood event per year is taken into account. A comparison of the two methods is presented in Sect. 6. Finally, our findings are discussed in Sect. 7. Further results and the derivation of our analytic results are provided in the Appendix.

\section{Methodology}

Our proposed methodology (illustrated by Fig. 1) is based on the combination of extreme value theory (Sects. 2.1, 2.2 and 2.3) and the concept of (stage-)damage functions (Sect. 2.4). Thus, we are able to calculate the expected annual damage (Sect. 2.5 and 2.6) within a considered region for different parameter scenarios. All employed symbols and their meanings are summarised in Table 1.

\subsection{Peak-over-threshold approach}

Extreme events are commonly characterised by employing extreme value theory (Coles, 2001; Hawkes et al., 2008). Besides the block maxima method, Peak-over-Threshold (POT) is a widely used approach (Coles, 2001), where the distribution of water levels, given that they exceed a certain threshold $u$, is estimated. Supposing the threshold $u$ to be high enough and assuming the independence of flood events, the excess water levels follow approximately a Generalised Pareto Distribution (GPD). In addition to $u$, the GPD is determined by a shape parameter $\xi$ and a scale parameter $\sigma$. Its cumulative distribution is of the following form:

$H_{u, \xi, \sigma}(x)= \begin{cases}1-\exp \left(-\frac{x-u}{\sigma}\right) & \text { if } \xi=0 \\ 1-\left(1+\xi \frac{x-u}{\sigma}\right)^{-1 / \xi} & \text { if } \xi \neq 0,\end{cases}$

with $x>u$. In the case $\xi<0$, the water level is bounded from above by a maximum possible water level $x_{\max }:=u-\sigma / \xi$ and we set $H_{u, \xi, \sigma}(x)=1$ for $x \geq x_{\max }$ accordingly. 
Table 1. Overview of employed symbols, their meanings, and section in which they are introduced.

\begin{tabular}{llc}
\hline Symbol & Meaning & Section \\
\hline$\xi$ & Shape parameter of the extreme value distribution & 2.1 \\
$\sigma$ & Scale parameter of the extreme value distribution & 2.1 \\
$u$ & Considered threshold of the extreme value distribution & 2.1 \\
$\mu$ & 1-year event; i.e. sea level that is exceeded on average once a year & 2.2 \\
$\Lambda$ & Intensity of the Poisson process; i.e. average number of sea level & 2.2 \\
& exceedances per year above $u$ & 2.4 \\
$\gamma$ & Exponent of the damage function & 5 \\
& Presumed protection level; i.e. maximum sea level against & \\
\hline
\end{tabular}

In our context, $u$ is the critical water level above which damage occurs or corresponds to the given protection height at the site. However, bearing in mind that $H_{u, \xi, \sigma}$ describes the limiting distribution of exceedances for an asymptotically increasing threshold, $u$ needs to be large enough to obtain a good approximation of the true distribution. In particular, if no protection is given and $u$ is freely chosen, a compromise between the adequacy of the statistical model (the larger $u$, the better the approximation) and the omission of smaller events $(x<u)$ needs to be found.

\subsection{Point process}

Section 2.1 provided the distribution of water levels given that the threshold $u$ is exceeded. However, an estimation of annual damage requires additional information on how often the sea level exceeds $u$. Therefore, we define a flood event as such an exceedance and use a point process to model the incidence of these events (Coles, 2001; Embrechts et al., 1997). By employing a Poisson process (Reiss and Thomas, 2007), the number of flood events $N$ within a specific year is Poisson-distributed with a certain mean value $\Lambda$, i.e.

$N \sim \operatorname{Poi}(\Lambda)$

Consequently, $\Lambda$ is the average number of flood events and $P(N=k)=\frac{\Lambda^{k}}{k !} \mathrm{e}^{-\Lambda}$ the probability of $k$ events within 1 year. The Poisson property Eq. (2) is a strong assumption, which is strictly valid for independent water levels and is commonly assumed in practice (e.g. Mudersbach and Jensen, 2010; Hawkes et al., 2008). The parameter $\Lambda$ can be estimated by counting the number of observed events divided by the corresponding time period. Furthermore, $\Lambda$ can be directly related to the GPD parameters: denoting $\mu$ as the 1year event, i.e. the water level that is exceeded on average once per year, it holds (Coles, 2001) that

$\Lambda= \begin{cases}\exp \left(-\frac{u-\mu}{\sigma}\right) & \text { if } \xi=0 \\ \left(1+\xi \frac{u-\mu}{\sigma-\xi(u-\mu)}\right)^{-1 / \xi} & \text { if } \xi \neq 0\end{cases}$

\subsection{Parameter effects}

We want to study the impact of sea level rise as well as potential protection measures on the flood damage. As illustrated in Fig. 2, two general effects can be observed within our framework. On the one hand, the frequency of flood events, i.e. the number of annual floods $N_{i}$, is expected to change. On the other hand, the intensities of occurring flood events can change, which would be represented in a change of the probability distribution of exceedances.

\subsubsection{Sea level rise}

We assume that a rise in mean sea levels results in a shift of today's sea level distribution towards higher water levels without deformation of the distribution (Kauker and Langenberg, 2000; Mudersbach et al., 2013). Other possible effects, e.g. a changing tide behaviour, can be modelled by a varying scale parameter $\sigma$ (see Appendix A). This scenario is illustrated in Fig. 2b where the time series is shifted by the mean sea level rise leading to increased numbers of flood events $N_{i}$ (which in turn change the parameter $\Lambda=\sum_{i=1}^{l} N_{i} / l$ ) and a modified probability distribution of exceedances. Accordingly, we adjust our model in such a way that every event of certain annuality in a particular year is increased by the corresponding sea level rise (McInnes et al., 2013). This is achieved by a simple modification of the parameters. Firstly, the frequency of exceedances will increase. Using the 1-year event $\mu$ as variable parameter, we consider a shift from $\mu$ to $\mu^{\prime}$ (i.e. a sea level rise of $\left(\mu^{\prime}-\mu\right) \mathrm{cm}$ ) and use Eq. (3) to derive the altered occurrence rate. Prior to this, also the scale parameter $\sigma$ of the exceedance distribution needs to be adjusted (Coles, 2001):

$\sigma^{\prime}=\sigma+\xi\left(\mu-\mu^{\prime}\right)$

which implies an altered width of the distribution in the case $\xi \neq 0$. These modifications result in a shift of each event with certain annuality by the magnitude of mean sea level rise. 

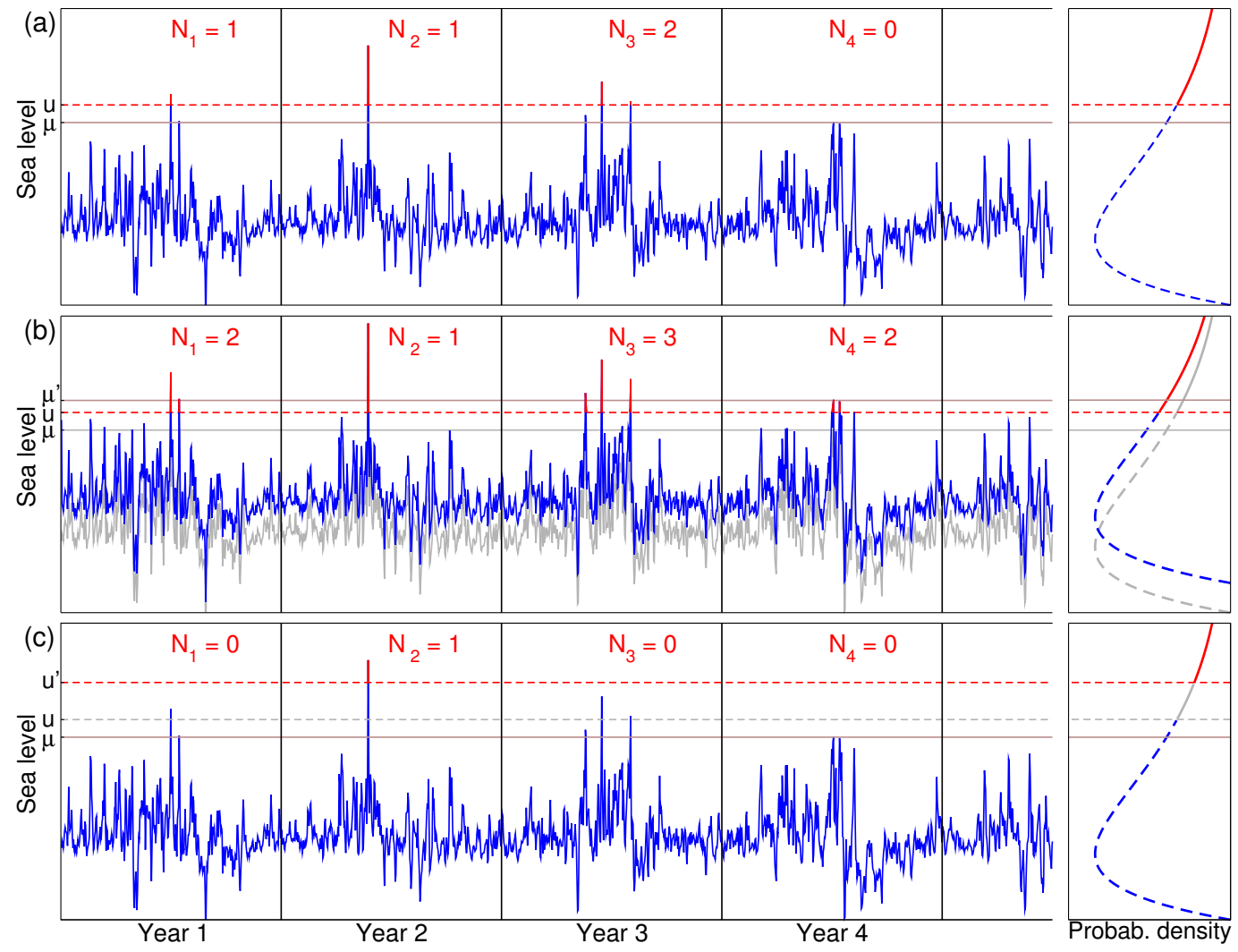

Figure 2. Illustrative time series of sea levels and their probability density function for several scenarios. (a) Current conditions with a threshold $u$ and the 1-year event $\mu$. (b) Increased mean sea level with a corresponding shift of the time series and thus the 1-year event from $\mu$ to $\mu^{\prime}$. (c) Supposing a protection height of $u^{\prime}$ implying an adjustment of the threshold from $u$ to $u^{\prime}$. The values $N_{1}, N_{2}, \ldots$ represent in all cases the number of exceedances within the corresponding year. The average value of the $N_{i}$ provides an estimator for occurrence rate $\Lambda$.

\subsubsection{Protection measures}

The implementation of a coastal protection measure will be considered in such a way that any damage from flood levels up to a specific protection height $\omega$ is avoided (as suggested by Hallegatte et al., 2013). Hence, we choose a new threshold $u^{\prime}=\omega$ representing the implemented protection height. Figure $2 \mathrm{c}$ illustrates this approach and it can be seen that the number of flood events $N_{i}$ as well as the probability distribution of exceedances changes if we raise the threshold from $u$ to $u^{\prime}$.

Given the GPD parameters with respect to a threshold $u$, the GPD distribution with respect to $u^{\prime}$ has the same shape parameter $\xi$ but a modified scale parameter $\sigma^{\prime}$ (see e.g. Katz et al., 2005):

$\sigma^{\prime}=\sigma+\xi\left(u^{\prime}-u\right)$.

Again, this also enters the derivation of a change in flood frequency using Eq. (3).

\subsection{Damage functions}

After having information about the occurrence of flood events, the resulting damage is obtained by means of a (stage-)damage function (Smith, 1994; Merz et al., 2010), which describes the correlations between the flood height and the corresponding damage (Fig. 1d).

Most commonly, damage functions are applied on the building scale (see e.g. Merz et al., 2010, and references therein). In our context, we use macroscopic damage functions (Boettle et al., 2011; Prahl et al., 2015), which provide the total damage within a spatially delineated region as a function of the maximum flood level. We assume that the form of such damage functions follows a power law (as suggested in Hallegatte et al., 2011; Boettle et al., 2011), i.e. for the damage caused by a flood of maximum height $x$ holds the proportionality

$F(x) \sim x^{\gamma}$.

See Boettle et al. (2013) for more details. In general the damage function exponent $\gamma$ is unknown, but values of 1.6 and 4.1 have been found for our case studies (Boettle et al., 2013). 


\subsection{Expected annual damage and uncertainty}

The combination of the methodologies above provides the probability distribution of the annual flood damage in a specific region (Fig. 1e). However, we restrict our investigations to the expectation value and the standard deviation of the annual damage. The annual damage $D$ is calculated as the sum of all single event damages $D_{i}$, i.e. $D=D_{1}+\cdots+D_{N}$, where $N \sim \operatorname{Poi}(\Lambda)$ is the number of flood events in the considered year. The Poisson property of $N$ implies for the expected number of flood events $\mathrm{E}_{N}=\Lambda$ and for its variance $\operatorname{Var}_{N}=\Lambda$. Using Wald's identities (Beichelt, 2006), the expected annual damage $\mathrm{E}_{D}$ and the standard deviation $\mathrm{SD}_{D}$ are

$\mathrm{E}_{D}=\Lambda \mathrm{E}_{D_{i}}$ and

$\mathrm{SD}_{D}=\left(\operatorname{Var}_{D}\right)^{1 / 2}=\left(\Lambda\left(\operatorname{Var}_{D_{i}}+\mathrm{E}_{D_{i}}^{2}\right)\right)^{1 / 2}$,

where $\mathrm{E}_{D_{i}}$ and $\operatorname{Var}_{D_{i}}$ describe the expected damage of a single flood event and its variance, respectively. Please note that all event magnitudes $D_{i}$ within 1 year are assumed to be independent and identically distributed (Coles, 2001).

\subsection{Computational calculations}

Given the extreme value parameters $u, \xi, \sigma, \Lambda$, and a damage function $F$, we calculate the expected annual damage $\mathrm{E}_{D}$ and the standard deviation $\mathrm{SD}_{D}$ by virtue of Eqs. (7) and (8). For this purpose, the required information on the single events $D_{i}$ is obtained via $\mathrm{E}_{D_{i}}=\int_{u}^{\infty} F(x) h_{u, \xi, \sigma}(x) \mathrm{d} x$ and $\mathrm{SD}_{D_{i}}^{2}=\int_{u}^{\infty}\left(\mathrm{E}_{D_{i}}-F(x)\right)^{2} h_{u, \xi, \sigma}(x) \mathrm{d} x$, where $h_{u, \xi, \sigma}(x)=$ $\frac{\mathrm{d}}{\mathrm{d} x} H_{u, \xi, \sigma}(x)$ is the probability density function of exceedances. From the computational perspective, the mentioned integrals need to be discretised and the upper limit replaced by a finite value $x_{\max }$. In the case $\xi<0$, the limit $x_{\max }$ represents the maximal possible water level as described in Sect. 2.1, otherwise it is set to such a high value that the resulting error becomes negligible. Partitioning the range of integration $\left[u, x_{\max }\right]$ by equidistant steps $\Delta x$ with $n$ midpoints $x_{1}, \ldots, x_{n}$, the following approximations are used:

$\mathrm{E}_{D_{i}} \approx \Delta x \sum_{j=1}^{n} F\left(x_{j}\right) h_{u, \xi, \sigma}\left(x_{j}\right)$ and

$\mathrm{SD}_{D_{i}}^{2} \approx \Delta x \sum_{j=1}^{n}\left(\mathrm{E}_{D_{i}}-F\left(x_{j}\right)\right)^{2} h_{u, \xi, \sigma}\left(x_{j}\right)$

\section{Sea level rise impacts}

We start in a general setting where the GPD parameters $\xi, \sigma$, $u$, as well as the damage function exponent $\gamma$ are given and investigate the behaviour of damage for rising sea levels.

All other parameters are kept constant in the following. As described in Sect. 2.3.1, we parametrise the mean sea level by the 1 -year event $\mu$. The following results are derived analytically (see Appendix B for details) and hold in an asymptotic sense. More precisely, the expected damage $\mathrm{E}_{D}$ divided by the provided expression, Eqs. (11)-(13), converges to a nonzero constant number for $\mu \rightarrow \infty$ (or, if it is bounded, by a value $\mu_{\max }$, for $\mu \rightarrow \mu_{\max }$ ). Hence, the following relations represent limit behaviours. Their practical use as approximations of the actual behaviour is examined in Sect. 4.

We find an increase of the annual damage by means of two separate effects (as described in Sect. 2.3.1): (i) higher frequency of events or (ii) higher severity of the events. Depending on the shape parameter $\xi$, three possible behaviours need to be distinguished:

(i) In the case $\xi=0$ (indicating an exponential tail in the sea level distribution), sea level rise leads to an exponentially increasing number of flood events while the alterations of single floods are negligible, overall implying an exponential dependence of the expected annual damage on the sea level:

$$
\mathrm{E}_{D}(\mu) \sim \mathrm{e}^{\mu / \sigma} .
$$

(ii) In contrast, we find a less steep relation if the water levels are bounded tailed (i.e. $\xi<0$ ):

$$
\mathrm{E}_{D}(\mu) \sim \mu^{\gamma-1 / \xi} \text {. }
$$

Here, the two effects are superposed: the average damage of an event increases with exponent $\gamma$ and the number of events with exponent $-1 / \xi$.

(iii) For the heavy-tailed case $(\xi>0)$, the damage can be characterised by a power law:

$\mathrm{E}_{D}(\mu) \sim\left(\mu_{\max }-\mu\right)^{-1 / \xi}$,

which holds for $\mu$ close to the maximum possible value $\mu_{\max }$. Approaching this value, the number of flood occurrences becomes very large and ends in a permanent flooding of the area under study for $\mu=\mu_{\max }$. As for $\xi=0$, this behaviour is solely caused by more frequent inundations and not by a changing severity of flood events.

It can be seen that each of the three possible relations involves a different set of parameters. Surprisingly, the damage function exponent $\gamma$ is not involved (in the case $\xi \geq 0$ ) or plays only a minor role (for $\xi<0$, the term $-1 / \xi$ in Eq. (12) is predominant for typical parameter values). This implies that the functional behaviour of $\mathrm{E}_{D}(\mu)$ is mostly independent of the determining factors of the damage function, such as the orography and the location of values in the case study area. In general, considering that $|\xi|$ typically takes small values, the expected damage increases super-linearly in all cases.

The expected annual damage only represents average values, and the actually occurring losses fluctuate considerably. 


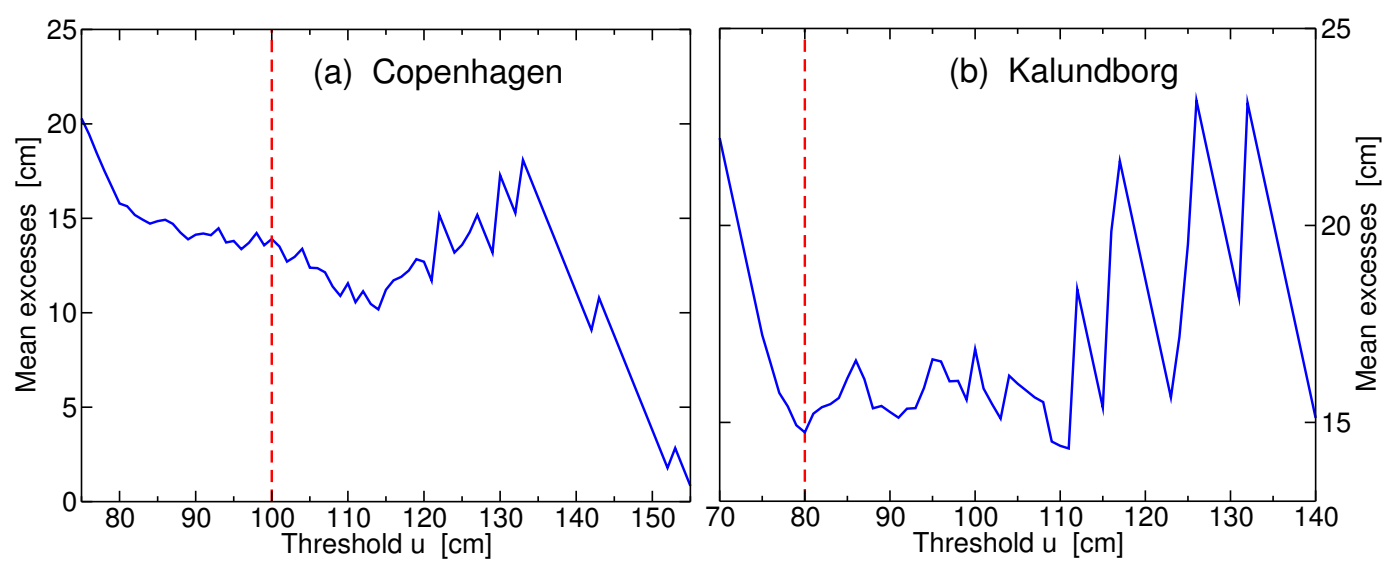

Figure 3. Average magnitudes of excesses over the threshold $u$ from available sea level records for varying thresholds in (a) Copenhagen and (b) Kalundborg. The dashed red lines represent the thresholds $u=100 \mathrm{~cm}$ (Copenhagen) and $u=80 \mathrm{~cm}$ (Kalundborg), which were used for our analysis.

Therefore, we also examine the uncertainty of our estimations by means of the standard deviation of the damage, $\mathrm{SD}_{D}$, and find expressions similar to the average value but with an additional factor of 0.5 in the exponents (see Appendix B for their derivations):

$$
\mathrm{SD}_{D}(\mu) \sim \begin{cases}\mathrm{e}^{0.5 \mu / \sigma} & \text { if } \xi=0 \text { (for large } \mu) \\ \mu^{\gamma-0.5 / \xi} & \text { if } \xi<0(\text { for large } \mu) \\ \left(\mu_{\max }-\mu\right)^{-0.5 / \xi} & \text { if } \left.\xi>0 \text { (for } \mu \text { close to } \mu_{\max }\right) .\end{cases}
$$

This uncertainty measure represents just a lower bound since it includes only the aleatory uncertainty from the fact that one does not know when the extremes occur and does not take into account additional epistemic uncertainties due to a lack of knowledge, e.g. stemming from the stage-damage relation (Merz et al., 2004) or the estimation of extreme value parameters (Hosking and Wallis, 1987). However, the relations imply that although the variability increases in all cases, the relative error of the estimate, $\mathrm{SD}_{D} / \mathrm{E}_{D}$, decreases with rising sea levels. Surprisingly, this implies that, in a sense, flood damage becomes more foreseeable.

Besides sea level rise, which is regarded as the main driver for higher and more frequent extremes (Menéndez and Woodworth, 2010), meteorological changes can play an important role. Evolving wind patterns, for instance, can lead to a modified distribution of water levels (Haigh et al., 2010), which in turn alters the damage distribution. Although this effect is not understood (Mudersbach and Jensen, 2010), the influence of a hypothetically changing scale parameter $\sigma$ on the damage is studied in Appendix B.

\section{Application}

We would like to illustrate how the respective variables behave in real examples and compare our analytic derivations from the previous section with numerical calculations (as described in Sect. 2.6) for two Danish case studies - namely the city of Copenhagen and the municipality of Kalundborg. The two locations were chosen due to the availability of damage functions as well as sea level records. Details on the case studies can be found in Hallegatte et al. (2011) and Boettle et al. (2011), respectively.

For the estimation of extreme value parameters in the two case studies, extreme sea level records from closely located gauges were preprocessed by subtracting a linear trend of $0.45 \mathrm{~cm}$ (Copenhagen) and $0.16 \mathrm{~cm}$ (Kalundborg) per year (derived from mean sea level data, available at http://www. psmsl.org). Next, a threshold $u$, above which the behaviour of water levels is modelled, needs to be chosen where a tradeoff between bias (low $u$ ) and variance (high $u$ ) is required. One necessary condition for an appropriate threshold $u$ is the linear dependence between the mean excesses and the thresholds close to $u$ (Coles, 2001). As can be seen in the mean excess plots in Fig. 3, this holds for thresholds around $100 \mathrm{~cm}$ (Copenhagen) and above $80 \mathrm{~cm}$ (Kalundborg), respectively. Bearing also in mind that a sufficient number of sea levels above the threshold is needed for the determination of parameters, the GPD parameters were estimated on the basis of the remaining 69 (Copenhagen) and 106 (Kalundborg) extreme sea levels. Using a maximum likelihood estimation (Embrechts et al., 1997), the parameters $\xi=-0.14$ and $\sigma=15.79 \mathrm{~cm}$ for Copenhagen and $\xi=0.08, \sigma=13.65 \mathrm{~cm}$ for Kalundborg were obtained. Since the damage function of Kalundborg shows only a negligible damage for sea levels below $140 \mathrm{~cm}$, the threshold was raised to $u=135 \mathrm{~cm}$, which entails a modified scale parameter of $\sigma=17.78 \mathrm{~cm}$ according to Eq. (5). Please note that this adjustment does not affect the stochastic accuracy and just avoids the consideration of negligible flood events. As the shape parameter $\xi$ is negative for Copenhagen, the possible sea levels are bounded and a maximum possible sea level of $x_{\max } \approx 215 \mathrm{~cm}$ is deduced. These parameters deviate from the previously performed estimation of GEV parameters based on annual maximum sea 

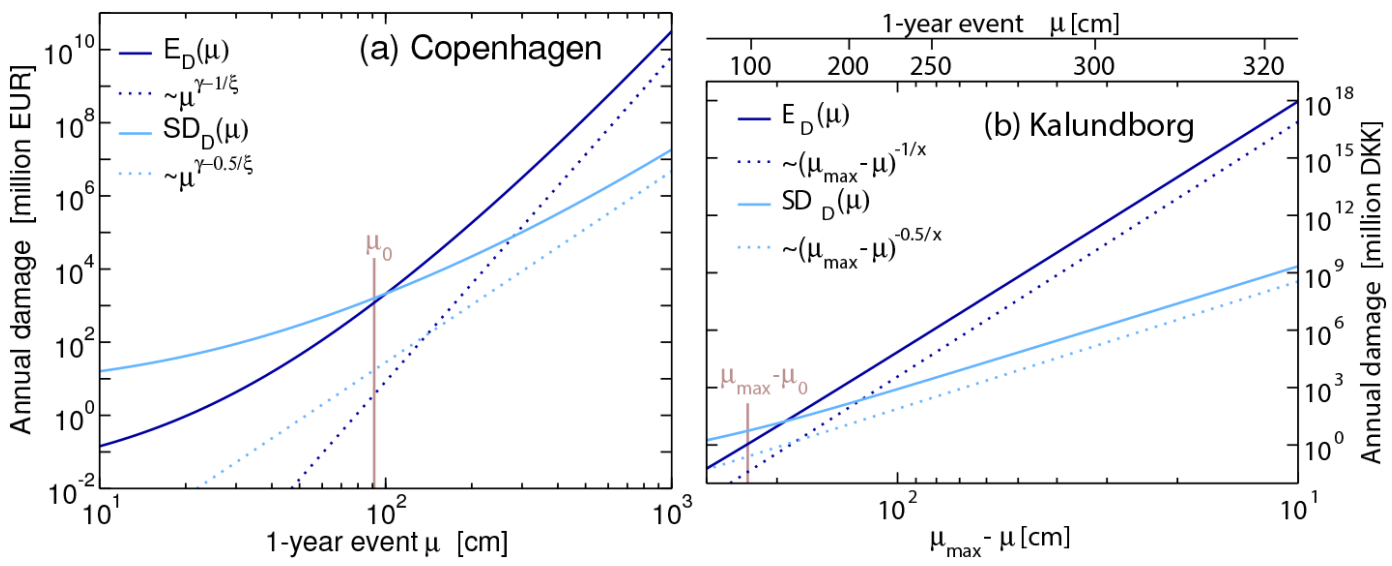

Figure 4. Expected annual damage (dark blue) and standard deviations (light blue) in (a) Copenhagen and (b) Kalundborg as a function of the mean sea level (parametrised by the 1-year flood $\mu$ ). The dotted lines show the asymptotic relations Eqs. (12), (13) and (14) with $\gamma=1.6$ and $\xi=-0.14$ (Copenhagen) and $\gamma=4.1$ and $\xi=0.08$ (Kalundborg). The values for the current 1 -year floods $\mu_{0}=91.21 \mathrm{~cm}($ Copenhagen) and $\mu_{0}=95.35 \mathrm{~cm}$ (Kalundborg) are indicated by brown vertical lines. The abscissa in the right panel is inverted and shows the difference between the 1-year flood $\mu$ and $\mu_{\max }=332.04 \mathrm{~cm}$ (at the top, the corresponding 1-year floods are displayed).

level records (Boettle et al., 2013). The occurrence rates $\Lambda$ for the description of the Poisson process were estimated by the average number of observed exceedances per year. These are $\Lambda=0.585$ (Copenhagen) and $\Lambda=0.083$ (Kalundborg). Finally, the 1-year events $\mu=91.21 \mathrm{~cm}$ (Copenhagen) and $\mu=95.35 \mathrm{~cm}$ (Kalundborg) were calculated by using Eq. (3).

The available damage functions support our presumption from Sect. 2.4 and follow roughly power laws with exponents $\gamma=1.6$ (Copenhagen) and $\gamma=4.1$ (Kalundborg). Further details on the damage functions can be found in Boettle et al. (2013). The exponent $\gamma$ is used for two purposes: (i) the parametrisation of the damage function needed for the functional description of sea level rise effects (Sect. 3) and (ii) for an extrapolation of the damage function beyond the provided range which in some cases is required for numerical calculations (namely, if $x_{\max }>10 \mathrm{~m}$, Sect. 2.6).

Once having this information, the annual damage can be calculated numerically (as described in Sect. 2.6) for varying mean sea levels parametrised by the 1 -year event $\mu$. Figure 4 shows this annual mean damage and its standard deviation as a function of $\mu$ and compares it with the asymptotic results. Since $\xi>0$ holds in the Kalundborg case, the parameter $\mu$ is bounded from above by a value $\mu_{\max }$ and we parametrise the damage by the difference $\mu_{\max }-\mu$. For $\mu$ approaching $\mu_{\max } \approx 332 \mathrm{~cm}$, the number of flood events becomes very large and ends in a permanent flooding of the considered region. It is likely that the municipality counteracts this tendency as soon as the occurring damage exceeds a tolerable value. Our projections are therefore explicitly based on a "no adaptation" scenario. We also disregard a modification of the damage function due to precedent damage and e.g. reduction of asset value. It can be seen that rising mean sea levels lead to an increase in expected damage and standard devi- ation, which is well described by our asymptotic results already for moderate values of $\mu$. This holds particularly for Kalundborg, but also in the case of Copenhagen - a similar shape of the dashed and solid lines can be detected. Overall, the asymptotic behaviours provide good estimates under the current conditions for both case studies. This shows that adequate projections of future flood damage can be obtained based on very few parameters. It needs to be highlighted again that in both cases the damage function exponent $\gamma$ plays only a minor role. While for Kalundborg the approximation is even independent from it, the asymptotic projection for Copenhagen, $\mu^{\gamma-1 / \xi} \approx \mu^{1.6+7.1}$, is clearly dominated by the shape parameter $\xi=-0.14$.

In practice, one is often interested in a temporal development of damage. In order to further elaborate on this issue, our approach requires a projection of mean sea levels. For the city of Copenhagen, such have been extracted from the Dynamical Interactive Vulnerability Assessment (DIVA) tool (Hinkel and Klein, 2003; Vafeidis et al., 2008) and we were thus able to study the damage as a function of time. Again, we suppose that changes in mean sea levels result in a shift of extreme events and add the estimated mean sea level rise to the corresponding events. Figure 5a displays the sea level projections for the SRES scenarios B1 (medium climate sensitivity) and A1B (high climate sensitivity) with a total rise of 11 and $26 \mathrm{~cm}$ by 2050 respectively. Panel b shows the resulting annual damage, exhibiting a steeper slope than the sea levels with an increase by a factor of roughly 2 (B1) and 4.6 (A1B) by 2050 , respectively.

\section{The effect of protection measures}

At this point, it is important to bear in mind that the severity of a flood disaster is not only determined by environmental 

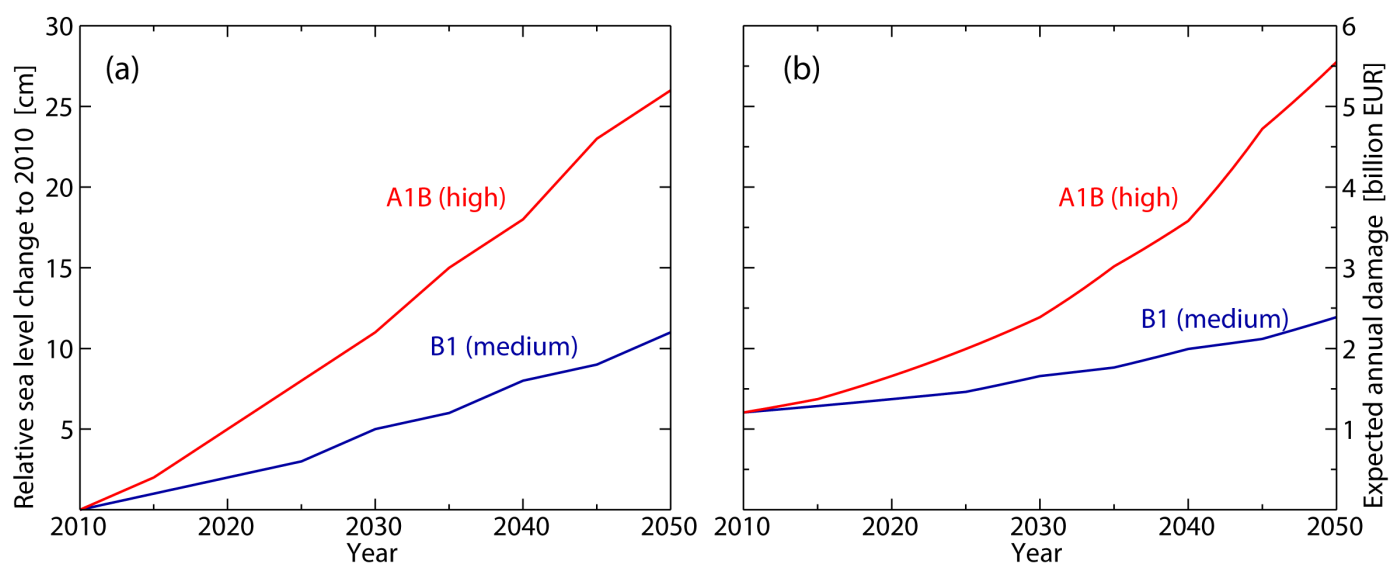

Figure 5. (a) Mean sea level projections for the SRES scenarios A1B (high climate sensitivity) and B1 (medium climate sensitivity) in Copenhagen provided by the DIVA tool (Hinkel and Klein, 2003; Vafeidis et al., 2008). (b) The expected annual damage as a function of time, based on the two scenarios.

factors but also to a significant extent by human decisions (Pielke Jr and Downton, 2000). In particular, the implementation of a flood defence measure can counteract the increasing flood risk (Fig. 1c). However, identifying the appropriate height of the protection measure is crucial for choosing a cost-efficient solution, i.e. an investment that pays off within a considered time period. Therefore, we investigate the effect of varying protection heights $\omega$ on the residual damage, assuming that inundations from flood levels below $\omega$ are completely avoided (as suggested by Hallegatte et al., 2013). Since the distribution of sea levels is bounded in the case $\xi<0$, the damage vanishes for a protection measure higher than the maximum possible water level $x_{\max }$. This is not the case for $\xi \geq 0$. In summary, we find the asymptotic relations

$$
\mathrm{E}_{D}(\omega) \sim \begin{cases}\omega^{\gamma} \mathrm{e}^{-\omega / \sigma} & \text { if } \xi=0 \text { (for large } \omega) \\ \left(x_{\max }-\omega\right)^{-1 / \xi} & \text { if } \left.\xi<0 \text { (for } \omega \text { close to } x_{\max }\right) \\ \omega^{\gamma-1 / \xi} & \text { if } \xi>0 \text { (for large } \omega) .\end{cases}
$$

As for rising sea levels, the behaviour of the expected damage depends fundamentally on the shape parameter $\xi$. While we find a decay that is dominated by an exponential component in the case $\xi=0$, a power law relation independent of the scale parameter $\sigma$ is found if $\xi>0$. For $\xi<0$, the expected damage follows a power law with the proximity of the protection height $\omega$ to the maximum water level $x_{\max }$. Remarkably, the expressions differ not only in their functional forms but also in the parameters involved. For instance, the exponent $\gamma$ of the damage function does not influence the behaviour in the case $\xi<0$ (as in Copenhagen). This highlights the decisive character of the shape parameter $\xi$, whose sign is not always unambiguous (Martins and Stedinger, 2000). Although a steep decrease in the damage is found in all cases, full flood safety can only be achieved if $\xi<0$, and a residual risk needs to be dealt with otherwise, even if potential protection failures, such as dyke breaches, are disregarded. Consid- ering the standard deviations, similar expressions are found (again with an additional factor of 0.5 in the exponents):

$$
\mathrm{SD}_{D}(\omega) \sim \begin{cases}\omega^{\gamma} \mathrm{e}^{-0.5 \omega / \sigma} & \text { if } \xi=0 \text { (for large } \omega \text { ) } \\ \left(x_{\max }-\omega\right)^{-0.5 / \xi} & \text { if } \left.\xi<0 \text { (for } \omega \text { close to } x_{\max }\right) \\ \omega^{\gamma-0.5 / \xi} & \text { if } \xi>0 \text { (for large } \omega \text { ) }\end{cases}
$$

Hence, in all cases, the relative variation of the damage, $\mathrm{SD}_{D} / \mathrm{E}_{D}$, grows with increasing protection levels. Consequently, damage in regions with high flood protection standards is subject to a wider range of relative uncertainty, indicating a higher contribution of low-probability high-impact events to the total damage (Merz et al., 2009). This shows that although coastal protection can reduce the average damage significantly, it cannot always avert the threat of very extreme floods.

Regarding the case studies Copenhagen and Kalundborg, Fig. 6 shows that the results from the numerical analyses of different protection levels can be very well approximated by our analytical relations from Eqs. (15) and (16). In contrast to the consideration of rising mean sea levels (Fig. 4), the Copenhagen case provides a better accordance than Kalundborg. This is due to the fact that in Copenhagen the parameter space of $\omega$ is limited by $x_{\max }$ and hence the asymptotic range of convergence is closer to the considered value. In any case, our results represent suitable estimations, which might be useful in planning and decision-making processes of coastal protection measures.

\section{Comparison with block maxima approach}

Beside the point process approach, the method of block maxima using the GEV distribution is a common approach in extreme value theory (Coles, 2001). Mathematically speaking, the GEV distribution is the limit distribution of properly normalised maxima of a sequence of independent and identically distributed random variables. In practice, it is used to 

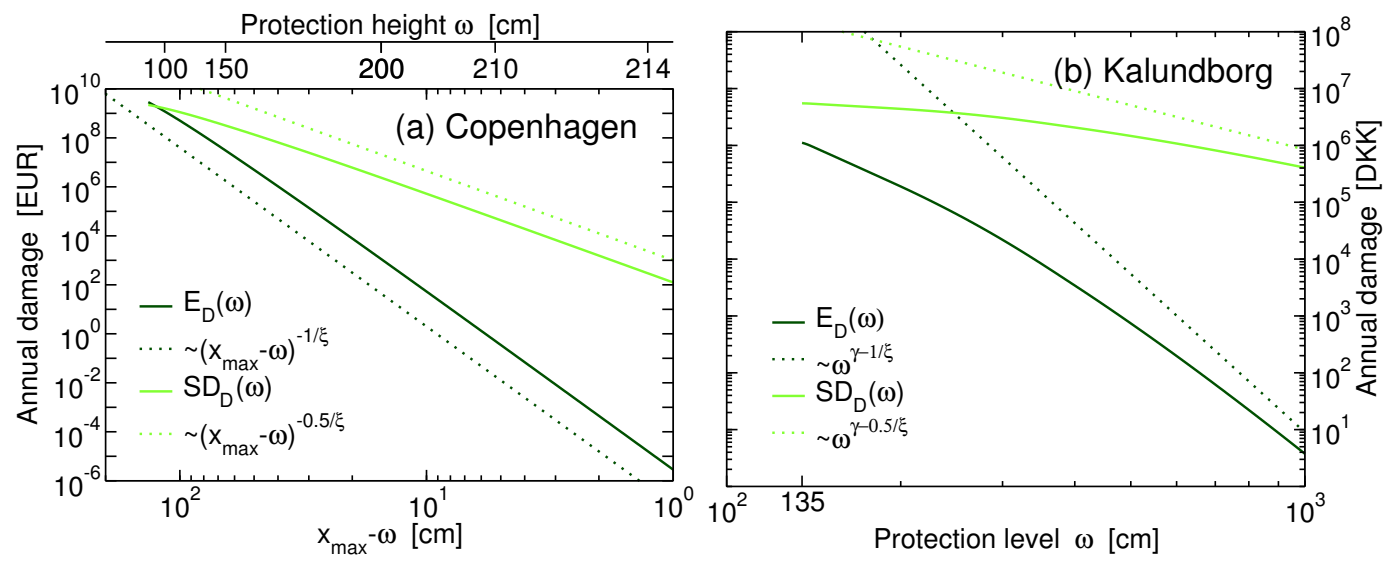

Figure 6. Expected annual damage (dark green) and standard deviations (light green) in (a) Copenhagen and (b) Kalundborg as a function of the protection level $\omega$. The abscissa in the left panel is inverted and shows the difference between the protection level $\omega$ and the maximum possible water level $x_{\max }=215.28 \mathrm{~cm}$ (at the top, the corresponding protection heights are displayed). Since no considerable damage occurs in Kalundborg for sea levels below $135 \mathrm{~cm}$, only protection levels above $\omega=135 \mathrm{~cm}$ are considered. The dotted lines follow the power laws from Eqs. (15) and (16) with the estimated damage function exponents $\gamma=1.6$ (Copenhagen) and $\gamma=4.1$ (Kalundborg).

estimate the distribution of the maximum value within a time window of a certain size (e.g. 1 year). Using the block maxima approach, only one flood event (the most severe) per year is considered, implying that all other events (i.e. the second, third, ... largest) are neglected. However, the point process approach and the block maxima approach are strongly interrelated (Coles, 2001; Katz et al., 2005). In particular, the parameters from the one approach can be easily derived from the other.

Complementary to the work in hand, an analogous analysis using the block maxima instead of the point process approach has been carried out recently (Boettle et al., 2013). Considering sea level rise, the asymptotic results of the two approaches differ significantly and a less steep increase in annual damage is found for arbitrary shape parameters $\xi$ if block maxima are considered. This is due to the fact that the average number of damage-causing floods per year increases and the omission of events in the block maxima approach takes effect. In the case that more than one flooding per year is expected, the point process method therefore represents the better choice as it adds significant information.

In contrast, an increasing variability in the sea levels, reflected in a changing scale parameter $\sigma$ (see Appendix B), leads to the same results for the two approaches. This can be explained by Eq. (3), which indicates that for an increasing scale parameter $\sigma$ the number of annual flood events converges to 1 . That is, on average there is one exceedance of the given threshold per year, which naturally coincides with the annual maximum sea level.

Finally, investigating increasing protection levels, the results of the two approaches again coincide. This is not surprising, since for high protection levels, inundations are very rare and more than one flooding per year is very unlikely. Consequently, the disregard of additional floods becomes negligible and the annual flood damage is typically determined by one - the most severe - flood event.

Both approaches are based on extreme value theory but differ in the extreme sea levels that are taken into account. Since the point process approach presented in this work is able to consider all relevant flood events, it can be considered as advantageous, particularly for the investigation of sea level rise impacts. However, the choice of the threshold $u$ is crucial and not always evident which makes the method more complex to apply. In general, as we have seen, the shape parameter $\xi$ is very decisive for the damage behaviour. Its determination is therefore of utmost importance and in case of doubt the method with a better data availability should be followed in order to guarantee the best possible estimation of $\xi$.

\section{Discussion}

Despite the accurate analytical formulation of the work at hand, some weaknesses need to be noted. For instance, the occurrence probability of a flood event on a specific day is assumed to be independent from the other days. In the shortterm there is a strong correlation between sea levels. This becomes apparent when considering the fact that storm surge events typically last for several days. In addition, it has been shown, that sea level records also comprise long-term correlations (Barbosa et al., 2006; Dangendorf et al., 2014). The clustering of extreme events (Eichner et al., 2007) might amplify the uncertainty. On the other hand, this is counteracted by the fact that two or more subsequent flood events (e.g. three sea level exceedances within 1 month) do not provide individual damages. That is, the actual damage is likely to be dominated by the first or highest of these events and will most likely not equal the sum of the damages corresponding 
to these events (given that they occur in a sufficiently long distance of time). In summary, although the presented approach still has some intrinsic errors, it overcomes the major shortcoming of a block maxima approach and hence can be considered as superior.

Studying the effect of sea level rise, we find that in any case the expected damage increases super-linearly with the mean sea level, when considering typical values of the shape parameter. This means that the losses always increase at a higher rate than the sea levels - a universal result that needs to be explored when the climate change impacts of sea level rise are discussed economically.

Our work also shows that the upcoming losses from sea level rise are mostly determined by the type of sea level extremes (i.e. the sign of the parameter $\xi$ ), which crucially dictates the power of $\mathrm{E}_{D}(\mu)$ (assuming constant coastal assets). This finding brings us to the following insights: (i) Since the steepness of the damage function (exponent $\gamma$ ) is mostly irrelevant, potential policies aiming at changing the slope of the damage function via relocation of valuable assets can reduce the expected losses, but a priori have only a marginal mitigation effect on the development of future flood damage. That is, such policies change the proportionality constant but hardly alter the proportionality. (ii) A reliable characterisation of sea level extremes is essential for a systematic assessment of climate change impacts due to sea level rise in the form of coastal floods. Thus, we plead for a high-resolution sea level network (Woodworth, 2010) if the losses from sea level rise are to be assessed on the regional or global scale.

In general, our results show how the complexity of climate change, adaptation, and flood damage can be disentangled by surprisingly simple and general expressions which are applicable to arbitrary regions and case studies. These relations are the basis for understanding the effect of sea level rise on coastal flood damage and are of great importance for the development of broad-scale assessment models in the context of climate change (e.g. Leimbach et al., 2010; Nordhaus and Yang, 1996).

The main text is complemented in two ways. Firstly, an additional analysis of flood damage in Copenhagen and Kalundborg as a function of the scale parameter $\sigma$ is provided in Appendix A. Finally, all expressions describing the asymptotic behaviours of the damage and its standard deviation are mathematically proven in Appendix B. 


\section{Appendix A: Further results}

In addition to varying the parameters $\mu$ and $\omega$ as discussed in the main text, the alteration of the scale parameter $\sigma$ represents also a potential impact from climate change. Such an effect could be explained by changing wind patterns leading to a lower or higher variability of water levels. Although alterations of $\sigma$ can be observed (Mudersbach and Jensen, 2010), the underlying mechanism behind is still unexplained. Nevertheless, for the sake of completeness, we investigate the hypothetical effect of a varying scale parameter $\sigma$ on the annual damage - analogous to the other parameter shifts. The corresponding asymptotic relations, Eqs. (B14) and (B15), are derived in the following section. Figure A1 illustrates the comparison of numerical calculations with the asymptotic results. It can be seen that in both case studies the increase of damage is less steep than the asymptotic behaviours for $\sigma$ close to the present value $\sigma_{0}$ and that a convergence is found for considerably larger values of $\sigma$.

\section{Appendix B: Analytical derivation of parameter effects}

In this section we derive the asymptotic relations from the main text. The section comprises three parts, each part considering the effects of changing parameters $\mu, \sigma$, as well as $\omega$ on one variable. First, in Sect. B1, we derive the effects of changing parameter values on the expected number of annual flood events. That is, how many threshold exceedances of the sea level can be expected within one year? Given such an exceedance, the magnitude of the sea level is still random with a certain probability distribution, from which a probability distribution of the corresponding damage can be derived. How this distribution alters with changing parameters is described in Sect. B2. Finally, combining the number of flood events and the damage of a single flood event provides the annual damage. The derivation of the relations for the expectation value and the standard deviation of the annual damage is presented in Sect. B3.

The provided expressions describe the damage for asymptotically large parameter values or, in case they are bounded, for parameters approaching their limit. This means that the numerically calculated values divided by the analytic result obtained converge to a non-zero constant number for increasing parameter values. In the whole section, the Generalised Pareto probability density function with regard to the threshold $u$, the shape parameter $\xi$, and the scale parameter $\sigma$ is denoted by $h_{u, \xi, \sigma}$.

\section{B1 Effects on the occurrence rate}

The investigation of the occurrence rate $\Lambda$ is based on Eq. (3). It has to be noted that shifting the parameter $\mu$ entails a modification of the scale parameter $\sigma$ by virtue of Eq. (4). In particular, the denominator in the case $\xi \neq 0$ is constant for varying $\mu$. One can see that for $\xi>0$ the parameter $\mu$ is bounded from above by a value $\mu_{\max }=\mu+\sigma / \xi$, at which $\Lambda$ becomes infinite. Accordingly, we study the asymptotic behaviour for $\mu$ approaching $\mu_{\max }$ in that case. Straightforward calculations provide the asymptotic relations for $\Lambda$ as a function of $\mu$ :

$$
\Lambda(\mu) \sim \begin{cases}\mathrm{e}^{\mu / \sigma} & \text { if } \xi=0 \text { (for large } \mu \text { ) } \\ \mu^{-1 / \xi} & \text { if } \xi<0 \text { (for large } \mu \text { ) } \\ \left(\mu_{\max }-\mu\right)^{-1 / \xi} & \text { if } \xi>0 \text { (for } \mu \text { close to } \mu_{\max } \text { ). }\end{cases}
$$

Considering a variable scale parameter $\sigma$, we obtain the relation

$\Lambda(\sigma) \sim 1$ (for large $\sigma$ ),

which holds for arbitrary values of $\xi$ and asymptotically large $\sigma$. In the last part of our analysis, we alter the protection height $\omega$, represented by setting the threshold to $u=\omega$, which also leads to a modification of the scale parameter $\sigma$. Using Eq. (5), we consider the occurrence rate for asymptotically increasing $\omega$ and obtain

$$
\Lambda(\omega) \sim \begin{cases}\mathrm{e}^{-\omega / \sigma} & \text { if } \xi=0 \text { (for large } \omega) \\ \left(x_{\max }-\omega\right)^{-1 / \xi} & \text { if } \left.\xi<0 \text { (for } \omega \text { close to } x_{\max }\right) \\ \omega^{-1 / \xi} & \text { if } \xi>0 \text { (for large } \omega),\end{cases}
$$

where $\omega$ is assumed to be below the maximum possible sea level $x_{\max }$ in the case $\xi<0$. If a protection level above this value is chosen, no inundation can occur and $\Lambda$ is 0 .

With these expressions, the frequency of events is fully described for large parameter values and, in combination with the following section, the behaviour of the annual damage is derived in Sect. B3. Note that Eq. (B1) holds only for small values of $\Lambda$ and that the results are therefore only valid for the corresponding parameter values (Embrechts et al., 1997). Otherwise, if $\Lambda$ becomes too large, the GPD is not an adequate estimation of the water levels.

\section{B2 Effects on the event damage distribution}

Not only the number of flood events is affected by evolving parameters. As described in Sect. 2.2, in the case of a flood, its magnitude follows a GPD, which in turn is modified by changing parameters. In the following, all integrals are integrated over the whole support of the corresponding density function. For reasons of simplicity, we omit all integral limits in the text. Furthermore, we assume the shape parameter $\xi$ to be small enough such that all integrals exist (Katz et al., $2002)$ - this is ensured for $\xi<1 / \gamma$ (expectation value) and $\xi<0.5 / \gamma$ (standard deviation). A divergence would imply an infinite variance or average value of the annual damage.

Theorem 1 ( $\mu$ relations). Let the water levels above a threshold $u$ follow a GPD with parameters $\xi$ and $\sigma$ and let us suppose a power damage function $F(x)=x^{\gamma}\left(\gamma \in \mathbb{R}^{+}\right)$. For the damage $D_{i}$ of a single event we obtain asymptotically

$$
\mathrm{E}_{D_{i}}(\mu) \sim \begin{cases}1 & \text { if } \xi=0(\text { for large } \mu) \\ \mu^{\gamma} & \text { if } \xi<0(\text { for large } \mu) \\ 1 & \text { if } \xi>0\left(\text { for } \mu \text { close to } \mu_{\max }\right)\end{cases}
$$



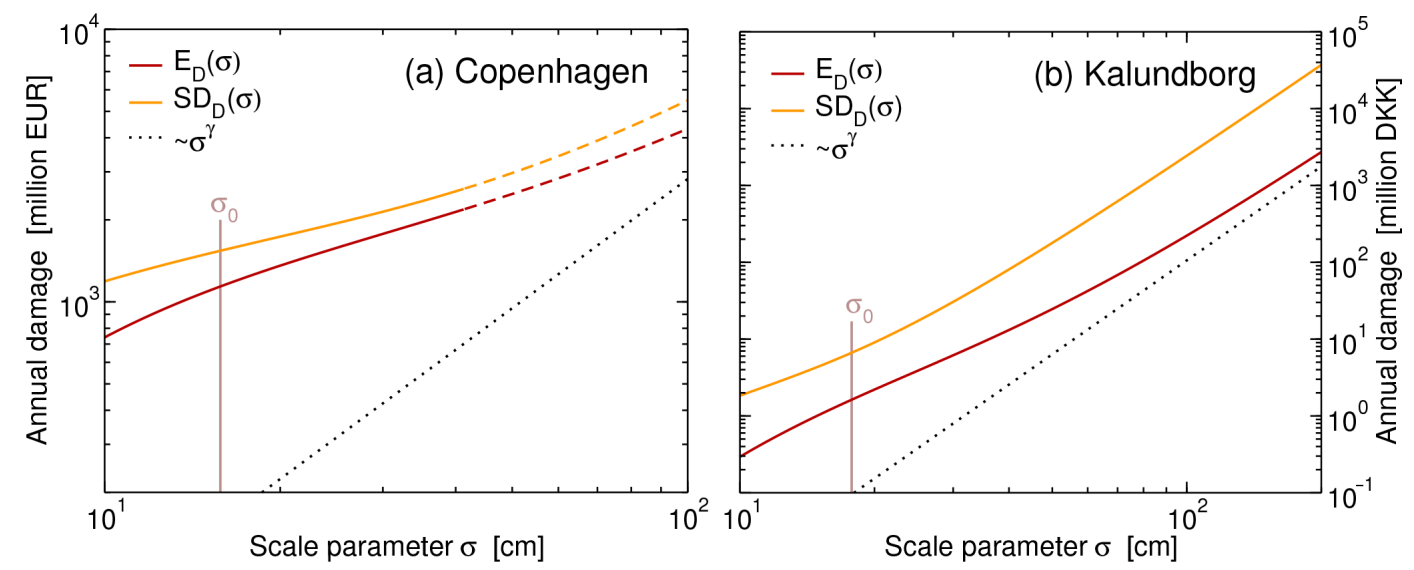

Figure A1. Expected annual damage (red) and standard deviations (orange) in (a) Copenhagen and (b) Kalundborg as a function of the scale parameter $\sigma$. The solid lines were numerically calculated with the available damage functions; the dashed continuations use an extrapolation of the damage function as a power law with exponent $\gamma=1.6$ (Copenhagen) and $\gamma=4.1$ (Kalundborg). The dotted line shows the asymptotic results from Eqs. (B14) and (B15) and the current values of the scale parameter $\sigma_{0}=15.79 \mathrm{~cm}\left(\right.$ Copenhagen) and $\sigma_{0}=17.78 \mathrm{~cm}$ (Kalundborg) are displayed as brown vertical lines.

and

$$
\mathrm{SD}_{D_{i}}(\mu) \sim \begin{cases}1 & \text { if } \xi=0(\text { for large } \mu) \\ \mu^{\gamma} & \text { if } \xi<0(\text { for large } \mu) \\ \mu_{\max }-\mu & \text { if } \xi>0\left(\text { for } \mu \text { close to } \mu_{\max }\right)\end{cases}
$$

with $\mu_{\max }:=\mu+\sigma / \xi$.

Proof. The relations for $\xi=0$ follow immediately from equations provided in Sect. 2.6. In the case $\xi<0$, a varying $\mu$ leads to a linear increase in $\sigma$ according to Eq. (4). Therefore, the relations are equivalent to $\mathrm{E}_{D_{i}}(\sigma) \sim \sigma^{\gamma}$ and $\operatorname{SD}_{D_{i}}(\sigma) \sim \sigma^{\gamma}$. The definition of the expectation value now provides

$$
\begin{aligned}
\mathrm{E}_{D_{i}}(\sigma) / \sigma^{\gamma}= & \int F(z+u / \sigma) h_{0, \xi, 1}(z) \mathrm{d} z \stackrel{\sigma \rightarrow \infty}{\longrightarrow} \\
& \int z^{\gamma} h_{0, \xi, 1}(z) \mathrm{d} z=\text { const. } \neq 0 .
\end{aligned}
$$

Furthermore, using the notation $m_{k}:=\int z^{k} h_{0, \xi, 1}(z) \mathrm{d} z$, we obtain

$$
\begin{aligned}
\left(\operatorname{SD}_{D_{i}}(\sigma) / \sigma^{\gamma}\right)^{2}= & \int F(z+u / \sigma)^{2} h_{0, \xi, 1}(z) \mathrm{d} z \\
& -\left(\int F(z+u / \sigma) h_{0, \xi, 1}(z) \mathrm{d} z\right)^{2} \\
& \stackrel{\sigma \rightarrow \infty}{\longrightarrow} m_{2 \gamma}-m_{\gamma}^{2}=\text { const. } \neq 0,
\end{aligned}
$$

which shows the asymptotic relations for $\xi<0$. In both cases we used uniform convergence to swap the integral and the limit.

As mentioned above, $\mu$ is bounded by $\mu_{\max }=\mu+\sigma / \xi$ in the case $\xi>0$ and we study the asymptotic behaviour for $\mu$ approaching $\mu_{\max }$. Considering $\mu \rightarrow \mu_{\max }$, Eq. (4) implies $\sigma \rightarrow 0$ and by using the uniform convergence of the integrand, it follows that

$\mathrm{E}_{D_{i}}(\mu)=\int F(\sigma z+u) h_{0, \xi, 1}(z) \mathrm{d} z \stackrel{\mu \rightarrow \mu_{\max }}{\longrightarrow} F(u)$,

which shows $\mathrm{E}_{D_{i}} \sim 1$ for $\mu$ approaching $\mu_{\max }$. In order to investigate the standard deviation for $\xi>0$, we make use of the Taylor expansion around $z=0$ :

$(\sigma z+u)^{\gamma}=u^{\gamma}+\gamma \sigma u^{\gamma-1} z+\gamma(\gamma-1) \sigma^{2} u^{\gamma-2} z^{2}+\mathcal{O}\left(\sigma^{3}\right)$. 
We obtain

$$
\begin{aligned}
\operatorname{Var}_{D_{i}}(\sigma)= & \int(\sigma z+u)^{2 \gamma} h_{0, \xi, 1}(z) \mathrm{d} z \\
& -\left(\int(\sigma z+u)^{\gamma} h_{0, \xi, 1}(z) \mathrm{d} z\right)^{2} \\
= & \int\left(u^{2 \gamma}+2 \gamma u^{2 \gamma-1} \sigma z+2 \gamma(2 \gamma-1)\right. \\
& \left.u^{2 \gamma-2} \sigma^{2} z^{2}+\mathcal{O}\left(\sigma^{3}\right)\right) h_{0, \xi, 1}(z) \mathrm{d} z \\
& -\left(\int \left(u^{\gamma}+\gamma u^{\gamma-1} \sigma z+\gamma(\gamma-1)\right.\right. \\
& \left.\left.u^{\gamma-2} \sigma^{2} z^{2}+\mathcal{O}\left(\sigma^{3}\right)\right) h_{0, \xi, 1}(z) \mathrm{d} z\right)^{2} \\
= & u^{2 \gamma}+2 \gamma u^{2 \gamma-1} \sigma m_{1}+2 \gamma(2 \gamma-1) \\
& u^{2 \gamma-2} \sigma^{2} m_{2}+\mathcal{O}\left(\sigma^{3}\right) \\
& -\left(u^{2 \gamma}+2 \gamma u^{2 \gamma-1} \sigma m_{1}+\gamma^{2} u^{2 \gamma-2} \sigma^{2} m_{1}^{2}\right. \\
& \left.+2 \gamma(\gamma-1) u^{2 \gamma-2} \sigma^{2} m_{2}+\mathcal{O}\left(\sigma^{3}\right)\right) \\
= & \underbrace{\text { const. }}_{\neq 0} \cdot \sigma^{2}+\mathcal{O}\left(\sigma^{3}\right)
\end{aligned}
$$

with the $k$ th moments $m_{k}:=\int z^{k} h_{0, \xi, 1}(z) \mathrm{d} z$. Considering that $\sigma$ converges linearly to 0 for $\mu \rightarrow \mu_{\max }$, it follows that $\operatorname{SD}_{D_{i}}(\sigma) / \sigma \rightarrow$ const. $\neq 0$ for $\sigma \rightarrow 0$ and in turn $\operatorname{SD}_{D_{i}}(\mu) \sim$ $\mu_{\max }-\mu$ for $\mu \rightarrow \mu_{\max }$.

Theorem 2 ( $\sigma$ relations). Let the water levels above a threshold $u$ follow a GPD with parameters $\xi$ and $\sigma$ and let us suppose a power damage function $F(x)=x^{\gamma}\left(\gamma \in \mathbb{R}^{+}\right)$. For the damage $D_{i}$ of a single flood event we obtain

$\mathrm{E}_{D_{i}}(\sigma) \sim \sigma^{\gamma}$ and $\operatorname{SD}_{D_{i}}(\sigma) \sim \sigma^{\gamma}$

for asymptotically large $\sigma$.

Proof. The proof corresponds to the case of increasing $\mu$ for $\xi<0$ in Theorem 1.

Finally, we derive expressions for the dependence on the protection height. As can be found in Coles (2001), a change of the threshold from $u$ to $u^{\prime}$ affects the scale parameter $\sigma$ by virtue of Eq. (5), which leaves the annualities of sea levels above the threshold unchanged.

Theorem 3 ( $\omega$ relations). Let the water levels above a threshold $u=\omega$ follow a GPD with parameters $\xi$ and $\sigma$, and let us suppose a power damage function $F(x)=x^{\gamma}$ $\left(\gamma \in \mathbb{R}^{+}\right)$. For the damage $D_{i}$ of a single event we obtain asymptotically

$$
\mathrm{E}_{D_{i}}(\omega) \sim \begin{cases}\omega^{\gamma} & \text { if } \xi=0(\text { for large } \omega) \\ 1 & \text { if } \xi<0\left(\text { for } \omega \text { close to } x_{\max }\right) \\ \omega^{\gamma} & \text { if } \xi>0(\text { for large } \omega)\end{cases}
$$

and

$$
\mathrm{SD}_{D_{i}}(\omega) \sim \begin{cases}\omega^{\gamma-1} & \text { if } \xi=0(\text { for large } \omega) \\ x_{\max }-\omega & \text { if } \xi<0\left(\text { for } \omega \text { close to } x_{\max }\right) \\ \omega^{\gamma} & \text { if } \xi>0(\text { for large } \omega),\end{cases}
$$

where $x_{\max }:=u-\sigma / \xi$ denotes the maximum possible sea level in the case $\xi<0$.

Proof. Let $u$ denote the current value of the threshold and $\omega$ the variable protection height corresponding to the new threshold $u^{\prime}$. We use Eq. (5) to calculate the scale parameter $\sigma^{\prime}$ which describes the excesses above the threshold $\omega=u^{\prime}$ and obtain

$$
\begin{aligned}
\mathrm{E}_{D_{i}}(\omega) & =\int F(x) h_{\omega, \xi, \sigma}(x) \mathrm{d} x \\
& =\int\left(\sigma^{\prime} z+\omega\right)^{\gamma} h_{0, \xi, 1}(z) \mathrm{d} z \\
& =\int(\sigma z+\xi z(\omega-u)+\omega)^{\gamma} h_{0, \xi, 1}(z) \mathrm{d} z .
\end{aligned}
$$

In the case $\xi \geq 0$, the uniform convergence of the integrand provides

$$
\begin{aligned}
\mathrm{E}_{D_{i}}(\omega) / \omega^{\gamma} & =\int(\xi z+1+z(\sigma-\xi u) / \omega)^{\gamma} h_{0, \xi, 1}(z) \mathrm{d} z \\
& \stackrel{\omega \rightarrow \infty}{\longrightarrow} \int(\xi z+1)^{\gamma} h_{0, \xi, 1}(z) \mathrm{d} z \\
& =\text { const. } \neq 0,
\end{aligned}
$$

which shows the asymptotic relation $\mathrm{E}_{D_{i}}(\omega) \sim \omega^{\gamma}$ for $\xi \geq 0$. For the corresponding standard deviation in the case $\xi=0$ it follows that

$$
\begin{aligned}
\operatorname{Var}_{D_{i}}(\omega)= & \int(\sigma z+\omega)^{2 \gamma} h_{0,0,1}(z) \mathrm{d} z \\
& -\left(\int(\sigma z+\omega)^{\gamma} h_{0,0,1}(z) \mathrm{d} z\right)^{2} .
\end{aligned}
$$

Using the Taylor expansion around $z=0$,

$$
\begin{aligned}
(\sigma z+\omega)^{\gamma}= & \omega^{\gamma}+\gamma \omega^{\gamma-1} \sigma z \\
& +\gamma(\gamma-1) / 2 \omega^{\gamma-2} \sigma^{2} z^{2}+\mathcal{O}\left(\omega^{\gamma-3}\right),
\end{aligned}
$$

we obtain 


$$
\begin{aligned}
\operatorname{Var}_{D_{i}}(\omega)= & \int\left(\omega^{2 \gamma}+2 \gamma \omega^{2 \gamma-1} \sigma z\right. \\
& +\gamma(2 \gamma-1) \omega^{2 \gamma-2} \sigma^{2} z^{2} \\
& \left.+\mathcal{O}\left(\omega^{2 \gamma-3}\right)\right) h_{0,0,1}(z) \mathrm{d} z \\
& -\left(\int \left(\omega^{\gamma}+\gamma \omega^{\gamma-1} \sigma z\right.\right. \\
& +\gamma(\gamma-1) / 2 \omega^{\gamma-2} \sigma^{2} z^{2} \\
& \left.\left.+\mathcal{O}\left(\omega^{\gamma-3}\right)\right) h_{0,0,1}(z) \mathrm{d} z\right)^{2}
\end{aligned}
$$

and straightforward calculations provide

$$
\begin{aligned}
\operatorname{Var}_{D_{i}}(\omega)= & \left(m_{2}-m_{1}^{2}\right) \gamma^{2} \sigma^{2} \omega^{2 \gamma-2} \\
& +\int \mathcal{O}\left(\omega^{2 \gamma-3}\right) h_{0,0,1}(z) \mathrm{d} z
\end{aligned}
$$

again using $m_{k}:=\int z^{k} h_{0,0,1}(z) \mathrm{d} z$. Now,

$$
\begin{aligned}
\lim _{\omega \rightarrow \infty} \operatorname{Var}_{D_{i}}(\omega) / \omega^{2 \gamma-2}= & \lim _{\omega \rightarrow \infty}\left(m_{2}-m_{1}^{2}\right) \gamma^{2} \sigma^{2} \\
& +\lim _{\omega \rightarrow \infty} \int \mathcal{O}\left(\omega^{-1}\right) h_{0,0,1}(z) \mathrm{d} z \\
= & \left(m_{2}-m_{1}^{2}\right) \gamma^{2} \sigma^{2} \\
= & \text { const. } \neq 0
\end{aligned}
$$

proves the expression for $\mathrm{SD}_{D_{i}}$ in the case $\xi=0$.

For $\xi>0$ it holds that

$$
\begin{aligned}
\operatorname{Var}_{D_{i}}(\omega) / \omega^{2 \gamma}= & \int \frac{1}{\omega^{2 \gamma}}(z(\sigma+\xi \omega-\xi u)+\omega)^{2 \gamma} \\
& h_{0, \xi, 1}(z) \mathrm{d} z \\
& -\left(\int \frac{1}{\omega^{\gamma}}(z(\sigma+\xi \omega-\xi u)+\omega)^{\gamma}\right. \\
& \left.h_{0, \xi, 1}(z) \mathrm{d} z\right)^{2} \\
& \stackrel{\omega \rightarrow \infty}{\longrightarrow} \int(\xi z+1)^{2 \gamma} h_{0, \xi, 1}(z) \mathrm{d} z \\
& -\left(\int(\xi z+1)^{\gamma} h_{0, \xi, 1}(z) \mathrm{d} z\right)^{2} \\
= & \text { const. } \neq 0,
\end{aligned}
$$

which proves the asymptotic relation $\operatorname{SD}_{D_{i}}(\omega) \sim \omega^{\gamma}$.

For the case $\xi<0$, we consider Eq. (B9):

$$
\begin{aligned}
\mathrm{E}_{D_{i}}(\omega) & =\int(\sigma z+\xi z(\omega-u)+\omega)^{\gamma} h_{0, \xi, 1}(z) \mathrm{d} z \\
& =\int\left(\xi z\left(\omega-x_{\max }\right)+\omega\right)^{\gamma} h_{0, \xi, 1}(z) \mathrm{d} z \\
& \stackrel{\omega \rightarrow x_{\max }}{\longrightarrow} \int x_{\max }^{\gamma} h_{0, \xi, 1}(z) \mathrm{d} z=x_{\max }^{\gamma},
\end{aligned}
$$

where we use the uniform convergence of the integrand to swap the integral and the limit. This proves the relation for
$\mathrm{E}_{D_{i}}$. In order to investigate the standard deviation, we define $\Delta \omega:=x_{\max }-\omega$ and examine the limit $\Delta \omega \rightarrow 0$. A Taylor expansion of $(\omega-\xi \Delta \omega z)^{\gamma}$ around $z=0$ provides

$$
\begin{aligned}
(\omega-\xi \Delta \omega z)^{\gamma}= & \omega^{\gamma}+\gamma \omega^{\gamma-1}(-\xi \Delta \omega) z \\
& +\gamma(\gamma-1) / 2 \omega^{\gamma-2}(-\xi \Delta \omega)^{2} z^{2} \\
& +\mathcal{O}\left(\Delta \omega^{3}\right)
\end{aligned}
$$

and for the variance it holds that

$$
\begin{aligned}
\operatorname{Var}_{D_{i}}(\omega)= & \int(\omega-\xi \Delta \omega z)^{2 \gamma} h_{0, \xi, 1}(z) \mathrm{d} z \\
& -\left(\int(\omega-\xi \Delta \omega z)^{\gamma} h_{0, \xi, 1}(z) \mathrm{d} z\right)^{2} \\
& \stackrel{\text { Eq. B } 10)}{=} \int\left(\omega^{2 \gamma}+2 \gamma \omega^{2 \gamma-1}(-\xi \Delta \omega) z\right. \\
& +2 \gamma(2 \gamma-1) / 2 \omega^{2 \gamma-2}(-\xi \Delta \omega)^{2} z^{2} \\
& \left.+\mathcal{O}\left(\Delta \omega^{3}\right)\right) h_{0}(z ; \xi, 1) \mathrm{d} z \\
& -\left(\int \left(\omega^{\gamma}+\gamma \omega^{\gamma-1}(-\xi \Delta \omega) z\right.\right. \\
& +\gamma(\gamma-1) / 2 \omega^{\gamma-2}(-\xi \Delta \omega)^{2} z^{2} \\
& \left.\left.+\mathcal{O}\left(\Delta \omega^{3}\right)\right) h_{0}(z ; \xi, 1) \mathrm{d} z\right)^{2} \\
= & \omega^{2 \gamma}-2 \gamma \omega^{2 \gamma-1} \xi \Delta \omega m_{1}+\gamma(2 \gamma-1) \\
& \omega^{2 \gamma-2} \xi^{2} \Delta \omega^{2} m_{2}+\mathcal{O}\left(\Delta \omega^{3}\right) \\
& -\left(\omega^{\gamma}-\gamma \omega^{\gamma-1} \xi \Delta \omega m_{1}+\gamma(\gamma-1) /\right. \\
& \left.2 \omega^{\gamma-2} \xi^{2} \Delta \omega^{2} m_{2}+\mathcal{O}\left(\Delta \omega^{3}\right)\right) \\
= & \omega^{2 \gamma}-2 \gamma \omega^{2 \gamma-1} \xi \Delta \omega m_{1}+\gamma(2 \gamma-1) \\
& \omega^{2 \gamma-2} \xi^{2} \Delta \omega^{2} m_{2}+\mathcal{O}\left(\Delta \omega^{3}\right) \\
& -\omega^{2 \gamma}+2 \gamma \omega^{2 \gamma-1} \xi \Delta \omega m_{1} \\
& -2 \gamma(\gamma-1) / 2 \omega^{2 \gamma-2} \xi^{2} \Delta \omega^{2} m_{2} \\
& -\gamma^{2} \omega^{2 \gamma-2} \xi^{2} \Delta \omega^{2} m_{1}^{2}+\mathcal{O}\left(\Delta \omega^{3}\right) \\
= & \gamma^{2} \omega^{2 \gamma-2} \xi^{2} \Delta \omega^{2} m_{2} \\
& -\gamma^{2} \omega^{2 \gamma-2} \xi^{2} \Delta \omega^{2} m_{1}^{2}+\mathcal{O}\left(\Delta \omega^{3}\right)
\end{aligned}
$$

and therefore

$$
\begin{aligned}
\operatorname{SD}_{D_{i}}(\omega) / \Delta \omega= & \left(\gamma^{2} \omega^{2 \gamma-2} \xi^{2} m_{2}-\gamma^{2} \omega^{2 \gamma-2} \xi^{2} m_{1}^{2}\right. \\
& +\mathcal{O}(\Delta \omega))^{1 / 2} \stackrel{\Delta \omega \rightarrow 0}{\longrightarrow} \text { const. } \neq 0
\end{aligned}
$$

which shows the statement of the theorem. 


\section{B3 Effects on the annual damage}

As stated in the main text, the total annual damage $D$ is calculated as the sum of all single event damages $D_{i}$, i.e. $D=D_{1}+\cdots+D_{N}$, where $N \sim \operatorname{Poi}(\Lambda)$ is the number of flood events in one year. This implies that $E_{N}=\operatorname{Var}_{N}=\Lambda$ and using Wald's identities (Beichelt, 2006), it follows

$\mathrm{E}_{D}=\Lambda \mathrm{E}_{D_{i}}$ as well as $\operatorname{Var}_{D}=\Lambda\left(\operatorname{Var}_{D_{i}}+\mathrm{E}_{D_{i}}^{2}\right)$

and together with the results from Sects. B1 and B2 we obtain

$$
\mathrm{E}_{D}(\mu) \sim \begin{cases}\mathrm{e}^{\mu / \sigma} & \text { if } \xi=0(\text { for large } \mu) \\ \mu^{\gamma-1 / \xi} & \text { if } \xi<0(\text { for large } \mu \text { ) } \\ \left(\mu_{\max }-\mu\right)^{-1 / \xi} & \text { if } \xi>0\left(\text { for } \mu \text { close to } \mu_{\max }\right)\end{cases}
$$

as well as

$$
\mathrm{SD}_{D}(\mu) \sim \begin{cases}\mathrm{e}^{0.5 \mu / \sigma} & \text { if } \xi=0(\text { for large } \mu) \\ \mu^{\gamma-0.5 / \xi} & \text { if } \xi<0(\text { for large } \mu) \\ \left(\mu_{\max }-\mu\right)^{-0.5 / \xi} & \text { if } \xi>0\left(\text { for } \mu \text { close to } \mu_{\max }\right)\end{cases}
$$

as asymptotic relations for varying mean sea levels. An altering scale parameter $\sigma$ leads to
$\mathrm{E}_{D}(\sigma) \sim \sigma^{\gamma}$ and

$\mathrm{SD}_{D}(\sigma) \sim \sigma^{\gamma}$

for asymptotically large values of $\sigma$ and for changing protection levels $\omega$ holds asymptotically

$$
\mathrm{E}_{D}(\omega) \sim \begin{cases}\omega^{\gamma} \mathrm{e}^{-\omega / \sigma} & \text { if } \xi=0 \text { (for large } \omega) \\ \left(x_{\max }-\omega\right)^{-1 / \xi} & \text { if } \left.\xi<0 \text { (for } \omega \text { close to } x_{\max }\right) \\ \omega^{\gamma-1 / \xi} & \text { if } \xi>0 \text { (for large } \omega)\end{cases}
$$

$$
\mathrm{SD}_{D}(\omega) \sim \begin{cases}\omega^{\gamma} \mathrm{e}^{-0.5 \omega / \sigma} & \text { if } \xi=0 \text { (for large } \omega) \\ \left(x_{\max }-\omega\right)^{-0.5 / \xi} & \text { if } \left.\xi<0 \text { (for } \omega \text { close to } x_{\max }\right) \\ \omega^{\gamma-0.5 / \xi} & \text { if } \xi>0 \text { (for large } \omega) .\end{cases}
$$

All results from the previous sections are summarised in Table B1. 
Table B1. The asymptotic behaviour of the number of annual flood events $\Lambda$, the expected damage from a single event $\mathrm{E}_{D_{i}}$ and the total annual damage $\mathrm{E}_{D}$ as well as the corresponding standard deviations $\mathrm{SD}_{D_{i}}$ and $\mathrm{SD}_{D}$, as functions of the 1-year event $\mu$ (with regard to a shift of all events), the scale parameter $\sigma$ and the protection level $\omega$. The values $\mu_{\max }=u+\sigma / \xi$ and $x_{\max }=\mu-\sigma / \xi$ represent upper limits for the parameters $\mu$ and $\omega$ in the case $\xi>0$ and $\xi<0$ respectively.

\begin{tabular}{|c|c|c|c|c|}
\hline & & \multicolumn{3}{|c|}{ Varying parameter } \\
\hline & & 1 -year event $\mu$ & Scale $\sigma$ & Protection height $\omega$ \\
\hline \multirow{3}{*}{$\Lambda$} & $\xi=0$ & $\sim \mathrm{e}^{\mu / \sigma}$ & $\sim 1$ & $\sim \mathrm{e}^{-\omega / \sigma}$ \\
\hline & $\xi<0$ & $\sim \mu^{-1 / \xi}$ & $\sim 1$ & $\omega \rightarrow x_{\max }\left(x_{\max }-\omega\right)^{-1 / \xi}$ \\
\hline & $\xi>0$ & $\mu \rightarrow \mu_{\max }\left(\mu_{\max }-\mu\right)^{-1 / \xi}$ & $\sim 1$ & $\sim \omega^{-1 / \xi}$ \\
\hline \multirow{3}{*}{$\mathrm{E}_{D_{i}}$} & $\xi=0$ & $\sim 1$ & $\sim \sigma^{\gamma}$ & $\sim \omega^{\gamma}$ \\
\hline & $\xi<0$ & $\sim \mu^{\gamma}$ & $\sim \sigma^{\gamma}$ & $\stackrel{\omega \rightarrow x_{\max }}{\sim} 1$ \\
\hline & $\xi>0$ & $\stackrel{\mu \rightarrow \mu_{\max }}{\sim} 1$ & $\sim \sigma^{\gamma}$ & $\sim \omega^{\gamma}$ \\
\hline \multirow{3}{*}{$\mathrm{E}_{D}$} & $\xi=0$ & $\sim \mathrm{e}^{\mu / \sigma}$ & $\sim \sigma^{\gamma}$ & $\sim \omega^{\gamma} \mathrm{e}^{-\omega / \sigma}$ \\
\hline & $\xi<0$ & $\sim \mu^{\gamma-1 / \xi}$ & $\sim \sigma^{\gamma}$ & $\omega \rightarrow x_{\max }\left(x_{\max }-\omega\right)^{-1 / \xi}$ \\
\hline & $\xi>0$ & $\stackrel{\mu \rightarrow \mu_{\max }}{\sim}\left(\mu_{\max }-\mu\right)^{-1 / \xi}$ & $\sim \sigma^{\gamma}$ & $\sim \omega^{\gamma-1 / \xi}$ \\
\hline \multirow{3}{*}{$\mathrm{SD}_{D_{i}}$} & $\xi=0$ & $\sim 1$ & $\sim \sigma^{\gamma}$ & $\sim \omega^{\gamma-1}$ \\
\hline & $\xi<0$ & $\sim \mu^{\gamma}$ & $\sim \sigma^{\gamma}$ & $\stackrel{\omega \rightarrow x_{\max }}{\sim} x_{\max }-\omega$ \\
\hline & $\xi>0$ & $\stackrel{\mu \rightarrow \mu_{\max }}{\sim} \mu_{\max }-\mu$ & $\sim \sigma^{\gamma}$ & $\sim \omega^{\gamma}$ \\
\hline \multirow{3}{*}{$\mathrm{SD}_{D}$} & $\xi=0$ & $\sim \mathrm{e}^{0.5 \mu / \sigma}$ & $\sim \sigma^{\gamma}$ & $\sim \omega^{\gamma} \mathrm{e}^{-0.5 \omega / \sigma}$ \\
\hline & $\xi<0$ & $\sim \mu^{\gamma-0.5 / \xi}$ & $\sim \sigma^{\gamma}$ & $\stackrel{\omega \rightarrow x_{\max }}{\sim}\left(x_{\max }-\omega\right)^{-0.5 / \xi}$ \\
\hline & $\xi>0$ & $\mu \rightarrow \mu_{\max }\left(\mu_{\max }-\mu\right)^{-0.5 / \xi}$ & $\sim \sigma^{\gamma}$ & $\sim \omega^{\gamma-0.5 / \xi}$ \\
\hline
\end{tabular}


Acknowledgements. We would like to thank Stéphane Hallegatte, Carlo S. Sørensen, and Jacob Arpe for the provision of data, as well as Luís Costa, Boris F. Prahl, and Dominik E. Reusser for fruitful discussions and comments. The research leading to these results has received funding from the European Community's Seventh Framework Programme under Grant Agreement No. 308497 (Project RAMSES). We also express our thanks to the Potsdam Research Cluster for Georisk Analysis, Environmental Change and Sustainability (PROGRESS) for their financial support.

Edited by: P. Tarolli

Reviewed by: G. Le Cozannet and one anonymous referee

\section{References}

Barbosa, S. M., Fernandes, M. J., and Silva, M. E.: Long-range dependence in North Atlantic sea level, Physica A, 371, 725-731, doi:10.1016/j.physa.2006.03.046, 2006.

Beichelt, F.: Stochastic Processes in Science, Engineering, and Finance, Chapman \& Hall/CRC, Boca Raton, FL, USA, 2006.

Boettle, M., Kropp, J. P., Reiber, L., Roithmeier, O., Rybski, D., and Walther, C.: About the influence of elevation model quality and small-scale damage functions on flood damage estimation, Nat. Hazards Earth Syst. Sci., 11, 3327-3334, doi:10.5194/nhess-113327-2011, 2011.

Boettle, M., Rybski, D., and Kropp, J. P.: How changing sea level extremes and protection measures alter coastal flood damages, Water Resour. Res., 49, 1199-1210, doi:10.1002/wrcr.20108, 2013

Coles, S.: An Introduction to Statistical Modeling of Extreme Values, Springer Series in Statistics, Springer, London, UK, 2001.

Dangendorf, S., Rybski, D., Mudersbach, C., Müller, A., Kaufmann, E., Zorita, E., and Jensen, J.: Evidence for long-term memory in sea level, Geophys. Res. Lett., 41, 5530-5537, doi:10.1002/2014GL060538, 2014.

Eichner, J. F., Kantelhardt, J. W., Bunde, A., and Havlin, S.: Statistics of return intervals in long-term correlated records, Phys. Rev. E, 75, 011128, doi:10.1103/PhysRevE.75.011128, 2007.

Embrechts, P., Klüppelberg, C., and Mikosch, T.: Modelling Extremal Events for Insurance and Finance, Applications of Mathematics, Springer, Berlin and Heidelberg, Germany, 1997.

Haigh, I., Nicholls, R., and Wells, N.: Assessing changes in extreme sea levels: application to the English Channel, 1900-2006, Cont. Shelf Res., 30, 1042-1055, doi:10.1016/j.csr.2010.02.002, 2010.

Hallegatte, S., Ranger, N., Mestre, O., Dumas, P., Corfee-Morlot, J., Herweijer, C., and Muir Wood, R.: Assessing climate change impacts, sea level rise and storm surge risk in port cities: a case study on Copenhagen, Climatic Change, 104, 113-137, doi:10.1007/s10584-010-9978-3, 2011.

Hallegatte, S., Green, C., Nicholls, R. J., and Corfee-Morlot, J.: Future flood losses in major coastal cities, Nature Clim. Change, 3, 802-806, doi:10.1038/nclimate1979, 2013.

Hawkes, P. J., Gonzalez-Marco, D., Sanchez-Arcilla, A., and Prinos, P.: Best practice for the estimation of extremes: a review, J. Hydraul. Res., 46, 324-332, doi:10.1080/00221686.2008.9521965, 2008.
Hinkel, J. and Klein, R. J. T.: DINAS-COAST: developing a method and a tool for dynamic and interactive vulnerability assessment, LOICZ Newsletter, 27, 1-4, 2003.

Hinkel, J., Lincke, D., Vafeidis, A. T., Perrette, M., Nicholls, R. J., Tol, R. S. J., Marzeion, B., Fettweis, X., Ionescu, C., and Levermann, A.: Coastal flood damage and adaptation costs under 21st century sea-level rise, P. Natl. Acad. Sci. USA, 111, 3292-3297, doi:10.1073/pnas.1222469111, 2014.

Hosking, J. R. M. and Wallis, J. F.: Parameter and Quantile Estimation for the Generalized Pareto Distribution, Technometrics, 29 , 339-349, doi:10.2307/1269343, 1987.

IPCC: in: Climate Change 2007: Impacts, Adaptation and Vulnerability, Contribution of Working Group II to the Fourth Assessment Report of the Intergovernmental Panel on Climate Change, edited by: Parry, M. L., Canziani, O. F., Palutikof, J. P., van der Linden, P. J., and Hanson, C. E., Cambridge University Press, Cambridge, UK and New York, NY, USA, 2007.

Katz, R. W., Parlange, M. B., and Naveau, P.: Statistics of extremes in hydrology, Adv. Water Resour., 25, 1287-1304, doi:10.1016/S0309-1708(02)00056-8, 2002.

Katz, R. W., Brush, G. S., and Parlange, M. B.: Statistics of extremes: modeling ecological disturbances, Ecology, 86, 11241134, doi:10.1890/04-0606, 2005.

Kauker, F. and Langenberg, H.: Two models for the climate change related development of sea levels in the North Sea - a comparison, Clim. Res., 15, 61-67, doi:10.3354/cr015061, 2000.

Leimbach, M., Bauer, N., Baumstark, L., and Edenhofer, O.: Mitigation costs in a globalized world: climate policy analysis with REMIND-R, Environ. Model. Assess., 15, 155-173, doi:10.1007/s10666-009-9204-8, 2010.

Martins, E. S. and Stedinger, J. R.: Generalized maximumlikelihood generalized extreme-value quantile estimators for hydrologic data, Water Resour. Res., 36, 737-744, doi:10.1029/1999WR900330, 2000.

McInnes, K. L., Macadam, I., Hubbert, G., and O'Grady, J.: An assessment of current and future vulnerability to coastal inundation due to sea-level extremes in Victoria, Southeast Australia, Int. J. Climatol., 33, 33-47, doi:10.1002/joc.3405, 2013.

Meehl, G. A., Hu, A., Tebaldi, C., Arblaster, J. M., Washington, W. M., Teng, H., Sanderson, B. M., Ault, T., Strand, W. G., and White, J. B.: Relative outcomes of climate change mitigation related to global temperature versus sea-level rise, Nature Clim. Change, 2, 576-580, doi:10.1038/nclimate1529, 2012.

Menéndez, M., and Woodworth, P. L.: Changes in extreme high water levels based on a quasi-global tide-gauge data set, J. Geophys. Res., 115, C10011, doi:10.1029/2009JC005997, 2010.

Merz, B., Kreibich, H., Thieken, A., and Schmidtke, R.: Estimation uncertainty of direct monetary flood damage to buildings, Nat. Hazards Earth Syst. Sci., 4, 153-163, doi:10.5194/nhess-4-1532004, 2004.

Merz, B., Kreibich, H., Thieken, A., and Schmidtke, R.: Estimation uncertainty of direct monetary flood damage to buildings, Nat Hazards Earth Syst. Sci., 4, 153-163, doi:10.5194/nhess-4-1532004, 2004

Merz, B., Kreibich, H., Schwarze, R., and Thieken, A.: Review article "Assessment of economic flood damage", Nat. Hazards Earth Syst. Sci., 10, 1697-1724, doi:10.5194/nhess-10-16972010, 2010. 
Mudersbach, C. and Jensen, J.: Nonstationary extreme value analysis of annual maximum water levels for designing coastal structures on the German North Sea coastline, J. Flood Risk Manag., 3, 52-62, doi:10.1111/j.1753-318X.2009.01054.x, 2010.

Mudersbach, C., Wahl, T., Haigh, I. D., and Jensen, J.: Trends in high sea levels of German North Sea gauges compared to regional mean sea level changes, Cont. Shelf Res., 65, 111-120, doi:10.1016/j.csr.2013.06.016, 2013.

Nicholls, R. J. and Cazenave, A.: Sea-level rise and its impact on coastal zones, Science, 328, 1517-1520, doi:10.1126/science.1185782, 2010.

Nicholls, R. J. and Tol, R. S. J.: Impacts and responses to sealevel rise: a global analysis of the SRES scenarios over the twenty-first century, Philos. T. R. Soc. A, 364, 1073-1095, doi:10.1098/rsta.2006.1754, 2006.

Nordhaus, W. D. and Yang, Z.: A regional dynamic generalequilibrium model of alternative climate-change strategies, Am. Econ. Rev., 86, 741-765, 1996.

Petherick, A.: Enumerating adaptation, Nature Clim. Change, 2, 228-229, doi:10.1038/nclimate1472, 2012.

Pielke Jr, R. A. and Downton, M. W.: Precipitation and damaging floods: trends in the United States, 1932-97, J. Climate, 13, 3625-3637, 2000.

Prahl, B. F., Rybski, D., Boettle, M., and Kropp, J. P.: Damage functions for climate-related hazards: unification and uncertainty analysis, Nat. Hazards Earth Syst. Sci. Discuss., 3, 6845-6881, doi:10.5194/nhessd-3-6845-2015, 2015.

Rahmstorf, S. and Coumou, D.: Increase of extreme events in a warming world, P. Natl. Acad. Sci. USA, 108, 17905-17909, doi:10.1073/pnas.1101766108, 2011.

Reiss, R.-D. and Thomas, M.: Statistical Analysis of Extreme Values, 3rd edn., Birkhäuser, Basel, Switzerland, 2007.
Seneviratne, S., Nicholls, N., Easterling, D., Goodess, C. M., Kanae, S., Kossin, J., Luo, Y., Marengo, J., McInnes, K., Rahimi, M., Reichstein, M., Sorteberg, A., Vera, C., and Zhang, X.: Changes in climate extremes and their impacts on the natural physical environment, in: Managing the Risks of Extreme Events and Disaster to Advance Climate Change Adaptation, A Special Report of Working Groups I and II of the Intergovernmental Panel on Climate Change (IPCC), edited by: Field, C. B., Barros, V., Stocker, T. F., Qin, D., Dokken, D. J., Ebi, K. L., Mastrandrea, M., Mach, K. J., Plattner, G.-K., Allen, S. K., Tignor, M., and Midgley, P., Cambridge University Press, Cambridge, UK, and New York, NY, USA, 109-230, 2012.

Smith, D. I.: Flood damage estimation - a review of urban stagedamage curves and loss functions, Water SA, 20, 231-238, 1994.

Tol, R. S. J.: Estimates of the damage costs of climate change Part II: Dynamic estimates, Environ. Resour. Econ., 21, 135-160, doi:10.1023/A:1014539414591, 2002.

Vafeidis, A. T., Nicholls, R. J., McFadden, L., Tol, R. S. J., Hinkel, J., Spencer, T., Grashoff, P. S., Boot, G., and Klein, R. J. T.: A new global coastal database for impact and vulnerability analysis to sea-level rise, J. Coastal Res., 24, 917-924, doi:10.2112/06-0725.1, 2008.

Woodworth, P. L.: A survey of recent changes in the main components of the ocean tide, Cont. Shelf Res., 30, 1680-1691, doi:10.1016/j.csr.2010.07.002, 2010.

Woodworth, P. L., Menéndez, M., and Gehrels, W. R.: Evidence for century-timescale acceleration in mean sea levels and for recent changes in extreme sea levels, Surv. Geophys., 32, 603-618, doi:10.1007/s10712-011-9112-8, 2011. 